\title{
Effects of long-term growth hormone therapy in a girl with Floating-Harbor syndrome
}

\author{
Hyun Woo Son, MD', \\ Jeong Eun Lee, MD', \\ Seung Hwan Oh, MD', \\ Changwon Keum ${ }^{3}$, \\ Woo Yeong Chung, MD, PhD ${ }^{1}$ \\ 'Department of Pediatrics, Inje Univer- \\ sity Busan Paik Hospital, Busan, Korea \\ ${ }^{2}$ Department of Diagnostic Laboratory \\ Medicine, Inje University Busan Paik \\ Hospital, Busan, Korea \\ ${ }^{3}$ Rare Genetic Disease Research Center, \\ 3 Billion, Seoul, Korea
}

\begin{abstract}
Floating-Harbor syndrome is a rare autosomal dominant disorder that presents with short stature, facial dysmorphism, significantly delayed bone age, skeletal abnormalities, speech and language problems, and intellectual disabilities. Although short stature is one of the main clinical manifestations, use of growth hormone therapy in Floating-Harbor syndrome patients has been limited. Only a few reports have investigated the response to growth hormone therapy with regard to final adult height. We report the case of a 7-year-old girl with FloatingHarbor syndrome and a heterozygous mutation, c.7330C > T (p.Arg2444*), in the $S R C A P$ gene. The patient exhibited dysmorphic facial features, severe intellectual disabilities, obsessive-compulsive and aggressive behaviors, and short stature without growth hormone deficiency. Her height standard deviation score improved after 55 months of growth hormone therapy.
\end{abstract}

Keywords: Floating-Harbor syndrome, Pediatric, Growth hormone, SRCAP

\section{Introduction}

Floating-Harbor syndrome (FHS; OMIM \# 136140) is a rare autosomal dominant disorder characterized by short stature with facial dysmorphism, significantly delayed bone age, skeletal abnormalities, speech and language problems, and intellectual disabilities. ${ }^{1)}$ Mutations in the Snf2-related CREBBP activator protein (SRCAP) gene have been identified as causes of FHS. ${ }^{2)}$ $S R C A P$ encodes the core catalytic component of the multiprotein chromatin remodeling SRCAP complex. Variable phenotypes of patients with FHS have been reported. ${ }^{3-6)}$ Most cases appear to be sporadic; however, autosomal dominant inheritance was reported in one familial case. ${ }^{7)}$ Although short stature is one of the main clinical manifestations, treatment with growth hormone $(\mathrm{GH})$ therapy in patients with FHS is rare. Only a few reports have explored the response to GH therapy with regard to final adult height. ${ }^{8)}$ We report a 4.5 -yeartreatment course with GH therapy in a Korean girl with FHS and a heterozygous c.7330C > T (p.Arg2444*) mutation in SRCAP.

Received: 29 March, 2019

Revised: 16 May, 2019

Accepted: 31 May, 2019

Address for correspondence: Woo Yeong Chung, MD, PhD

Department of Pediatrics, Inje University Busan Paik Hospital, Inje University College of Medicine, 75 BokJi-ro, Busanjin-gu, Busan 47392, Korea

Tel: +82-51-890-6280

Fax: +82-51-895-7785

E-mail: chungwy@chol.com

https://orcid.org/0000-0002-50247285

\section{Case report}

A 7-year-old girl with distinct facial features and short stature visited our hospital for diagnostic evaluation. She had been born via cesarean section at 36-week gestation without perinatal problems; her birth weight was $2.7 \mathrm{~kg}$ (25th-50th percentile). She was the second child of nonconsanguineous, healthy parents. Her midparental height was $160 \mathrm{~cm}$ (father's height, $180 \mathrm{~cm}$; mother's height, $153 \mathrm{~cm}$ ). Her elder brother exhibited normal growth and psychomotor development. There was no family history of genetic diseases.

At initial presentation, the patient's height was $105.8 \mathrm{~cm}(-3.54$ standard deviation score [SDS]); her weight was $16.3 \mathrm{~kg}(-2.45 \mathrm{SDS})$, and her head circumference was $51 \mathrm{~cm}(-0.3$ SDS). Her bone age was 4.5 years. She had dysmorphic facial features, which included a small, 
triangular face with deep-set eyes, a broad and bulbous nose, a short philtrum, and thin lips as well as hand anomalies, such as clinodactyly and a short thumb with clubbing. X-rays revealed middle phalange dysplasia and genu varum (Fig. 1).

The patient's fine motor and gross motor development were appropriate for her chronological age. She demonstrated severe intellectual disabilities as well as obsessive-compulsive and aggressive behaviors. The patient attended a special-education school and had been receiving rehabilitation treatment for the previous 2 years. Electroencephalogram, brain magnetic resonance imaging, echocardiography, and ultrasound of the kidneys were normal. Chromosome analysis indicated a 46, XX karyotype. Ophthalmological examination revealed strabismus and mild diplopia. Results of blood biochemical and urine studies were within the normal ranges. Thyroid function test results were also normal. Serum insulin-like growth factor 1 (IGF-1) and IGF-binding protein 3 levels were $223.1 \mathrm{ng} / \mathrm{mL}$ (normal range, $26-320 \mathrm{ng} / \mathrm{mL}$ ) and $2481.1 \mathrm{ng} / \mathrm{mL}$ (normal range, $2,188-4,996 \mathrm{ng} / \mathrm{mL}$ ), respectively. The $\mathrm{GH}$ peak levels provoked by insulin and L-dopa were $44.30 \mathrm{ng} / \mathrm{mL}$ and $6.07 \mathrm{ng} /$ $\mathrm{mL}$, respectively.

Whole-exome sequencing was performed to search for causative genes. Previous Sanger sequencing of single target genes and panel gene tests regarding the phenotypes of short

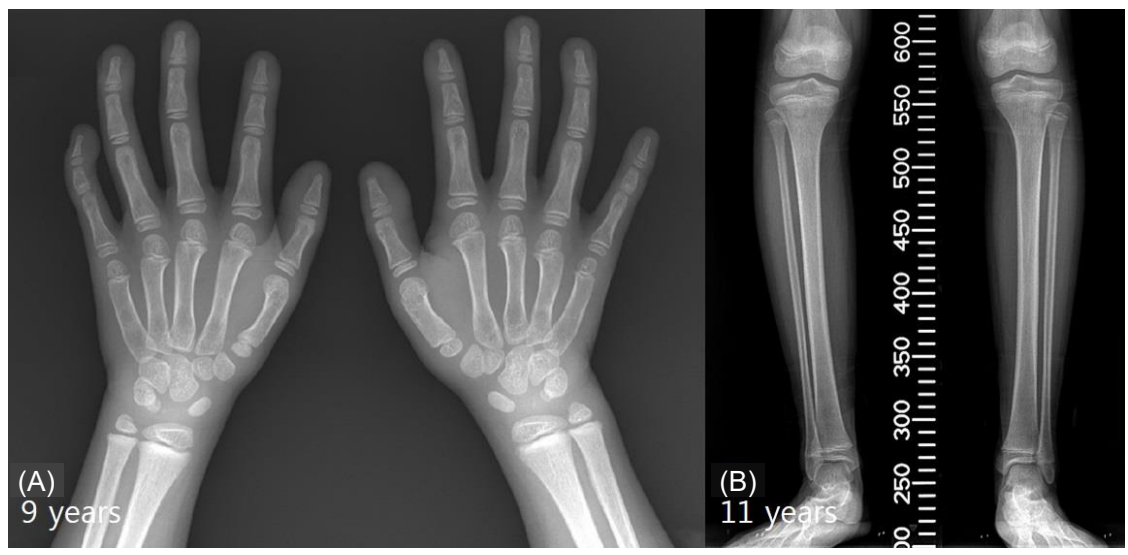

Fig. 1. (A) An X-ray of the hands of a 9-year-old patient displaying Floating-Harbor syndrome characteristics. Middle phalangeal hypoplasia, clinodactyly of the fifth finger, and hypoplastic metacarpals of the first finger are noted in both hands. Absence of a lunate and a hypoplastic scaphoid are shown bilaterally. (B) An X-ray of the legs of an 11-year-old patient with Floating-Harbor syndrome. Genu varum can be observed in the lower extremities bilaterally.
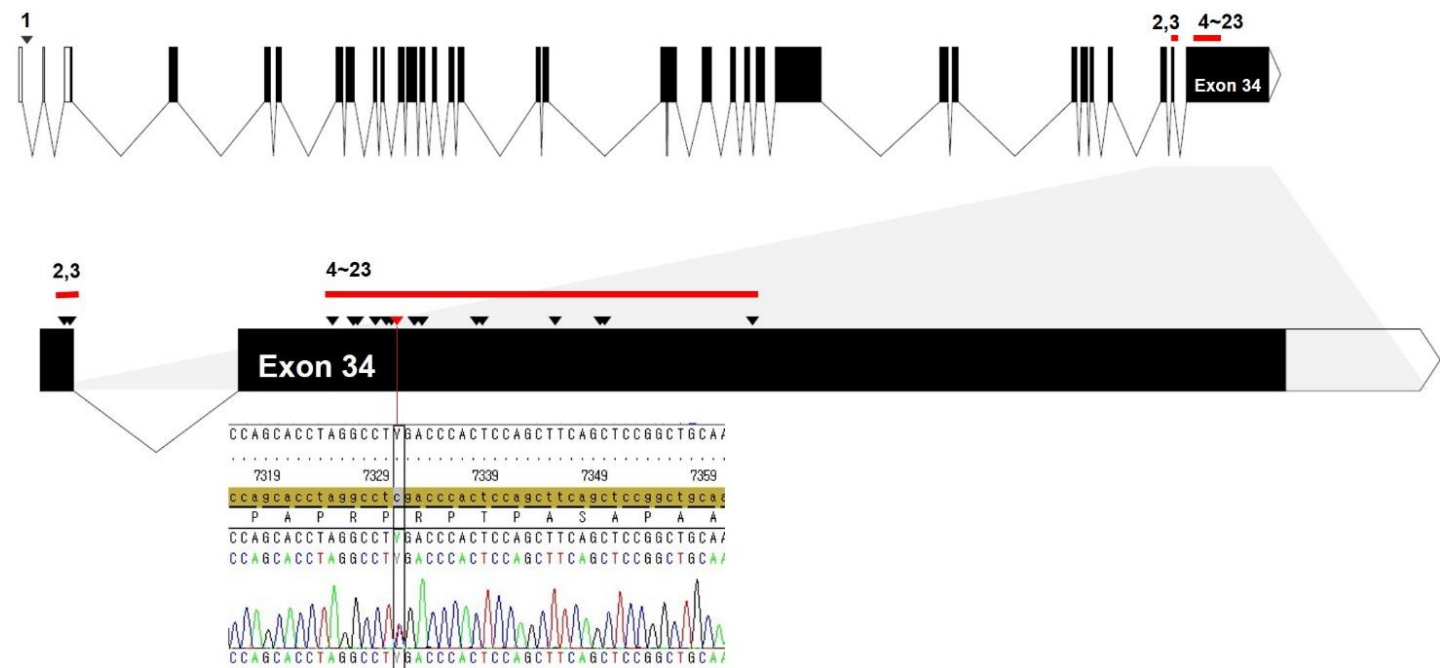

Fig. 2. A schematic structure of the SRCAP gene and the locations of mutations detected in Floating-Harbor syndrome patients. All mutations (arrowhead) were truncating and located on exons 33 and 34 except for a single splicing mutation (No. 1) located on the first intron. Arrowheads denote mutation sites of all cases in the literature (No. 1-23 listed in Table 1) according to the order of genomic sequences. Red bars indicate the clustered region of mutations. The specific mutation (c.7330C > T, p.Arg2444*) of the patient in this study was located at a mutational hotspot (exon 34, red arrowhead). 
stature and skeletal dysplasia presenting with intellectual disabilities revealed no pathogenic variants.

Genomic DNA was extracted from the buccal mucosa. The library was prepared to capture all exon regions of all human genes $(\sim 22,000)$ using the SureSelect kit (Agilent Technologies, Santa Clara, CA, USA). The captured regions were sequenced using the Illumina platform (NovaSeq, Illumina, Inc., San Diego, CA, USA). Raw genome sequencing data analysis, including alignment to the reference sequence (original GRCh37 from NCBI, February 2009), was performed with a mean depth of coverage of $100 \times(10 \times=99.2 \%)$. Variant calling, annotation, and prioritization were performed as previously described. ${ }^{9}$ Variants with minor allele frequencies $<0.05 \%$ for dominant disease association or $2 \%$ for recessive disease association were analyzed using population genome databases, such as the 1,000 genomes site (http://phase3browser.1000genomes.org), the Exome Variant Server (http://evs.gs.washington.edu/EVS/), and

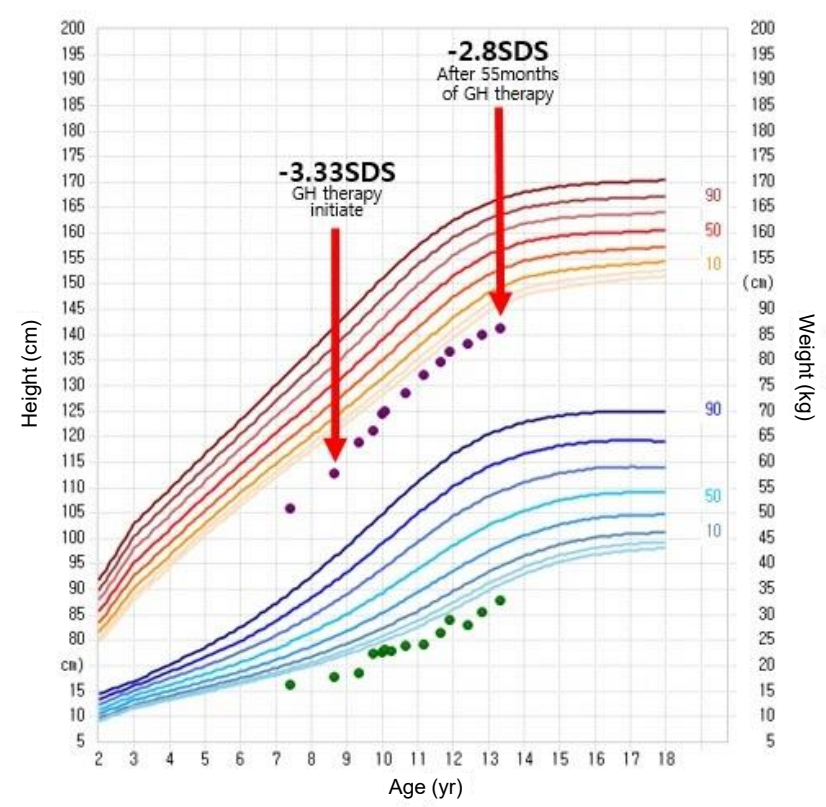

Fig. 3. Growth curve of our Floating-Harbor syndrome patient during growth hormone therapy. The red arrows indicate when our patient began growth hormone therapy, and the results were achieved after 55 months of treatment. $\mathrm{GH}$, growth hormone; SDS, standard deviation score.
ExAC (http://exac.broadinstitute.org/).

A heterozygous c.7330C $>\mathrm{T}\left(\mathrm{p} . \operatorname{Arg} 2444^{*}\right)$ mutation in $S R C A P$ was identified and confirmed by Sanger sequencing (Fig. 2). There were no pathogenic variations in other genes related to Rubinstein-Taybi syndrome, Shprintzen-Goldberg syndrome, or other short-stature syndromes, such as those linked to mutations in the CREBBP, EP300, SKI, and ACAN genes; coverage rates greater than 20X of exons and intronic flanking regions (20 bp) were $87 \%, 95.6 \%, 93.7 \%$, and $94.8 \%$, respectively.

At 8 years old, the patient's height was $112.8 \mathrm{~cm}(-3.33 \mathrm{SDS})$. Recombinant human GH replacement therapy was initiated $(1.0 \mathrm{U} / \mathrm{kg} / \mathrm{wk})$. After 55 months of GH therapy, her height was $141.3 \mathrm{~cm}(-2.7 \mathrm{SDS})$, and she showed a modest improvement in growth velocity of $6.2 \mathrm{~cm} / \mathrm{yr}$ (Fig. 3). Her bone age was consistent with her chronological age by 12 years (Fig. 4), at which point her Tanner stage was breast III. No adverse events were observed during $\mathrm{GH}$ treatment.

\section{Discussion}

We identified a heterozygous mutation on exon 34 of the SRCAP gene (c.7330C > T, p.R2444*) in a female Korean patient with FHS. This mutation has the highest frequency rate among FHS patients. Skeletal defects that result in altered height and posture and delayed bone age are typical of this syndrome, along with difficulties in communication and processing. ${ }^{1)}$ Other identifiable traits include dysmorphic facial features as described for our patient; however, these features can be subjective and nonspecific, complicating accurate clinical diagnosis of FHS.

SRCAP is located on chromosome 16p11.2 and includes 34 exons. SRCAP encodes an SNF2-related chromatin that remodels ATPase, which is a co-activator of CREB-binding protein (CREBBP). ${ }^{10)}$ The CREBBP gene and its homolog, E1Abinding protein $\mathrm{p} 300$, play an important role in regulating cell growth and development. A mutation within CREBBP causes Rubinstein-Taybi syndrome. Therefore, the clinical features of Rubinstein-Taybi syndrome and FHS overlap. Budisteanu et al. ${ }^{11)}$ proposed a checklist of clinical features suggestive of FHS based on the main clinical features reported in the literature. Characteristic facial features and delayed language abilities are

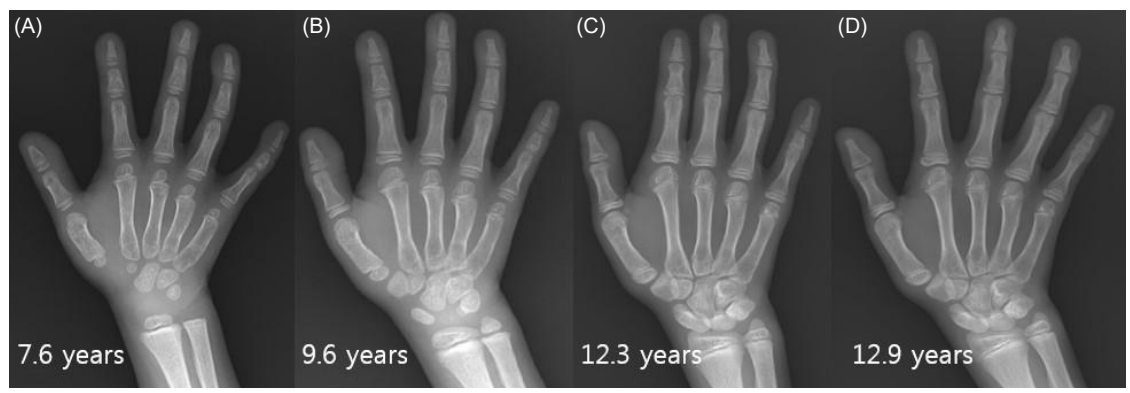

Fig. 4. Bone age progression in our Floating-Harbor syndrome patient during growth hormone therapy. Changes in bone age at the chronologic age of 7.6 years (A), 9.6 years (B), 12.3 years (C), and 12.9 years (D). 
mandatory for diagnosis of FHS, while short stature, delayed bone age, skeletal anomalies, and intellectual disabilities are frequent features. Other clinical manifestations, such as behavioral problems, eye anomalies, ear anomalies, dental issues, genitourinary malformations, cardiac malformations, gastrointestinal features, seizures, and hypothyroidism, are recurrent or infrequent. Because the 2 main features of FHS, facial dysmorphism and language delay, are nonspecific, variable phenotypes exist in FHS patients. It is difficult to clinically differentiate other genetic short stature syndromes that have overlapping clinical phenotypes. Table 1 shows all the mutations associated with FHS that have been identified in the literature, including those from this study, which have been categorized according to mutation site.
Choi et al. ${ }^{12)}$ reported the first Korean case of FHS with a novel mutation (c.7732dupT, p.Ser2578Phefs ${ }^{\star} 6$ ) in SRCAP. The patient was a 7-year-old boy, and his height was $113 \mathrm{~cm}$ (10th-25th percentile). His language was impaired, and he had a unique facial morphology.

Short stature is the most characteristic feature of FHS; however, there are limited data on the GH-IGF-1 axis in FHS. Defects in GH secretion or activity usually do not contribute to prenatal growth inhibition, whereas IGF-1 secretion or activity is associated with prenatal growth inhibition and microcephaly. Birth size is usually normal in patients with FHS, and growth velocity begins to slow during infancy. Garcia et al. ${ }^{13)}$ suggested that FHS may lead to impaired IGF-1 signaling because of the discrepancy between the modest growth response to GH

Table 1. Mutations associated with Floating-Harbor syndrome in the literature and in this study, categorized by mutation site

\begin{tabular}{|c|c|c|c|c|c|c|}
\hline No. & Mutation Site & Nucleotide changes & Amino acid changes & HGMD accession $^{\dagger}$ & $\mathrm{dbSNP}$ & References \\
\hline 1 & Intron 1 & c. $306+5 G>A$ & Splicing variant & CS177972 & NS & 15 \\
\hline 2 & Exon 33 & c. $6985 C>T$ & p.Arg2329* & CM1411785 & NS & 16 \\
\hline 3 & Exon 33 & c. $7000 C>T$ & p.Gln2334* & CM136741 & rs587777656 & 17 \\
\hline 4 & Exon 34 & c. $7165 G>T$ & p.Glu2389* & CM136742 & NS & 5 \\
\hline 5 & Exon 34 & c.7218_7219delTC & p.GIn2407Argfs*35 & CD121175 & rs199469467 & 3 \\
\hline 6 & Exon 34 & c.7218dupT & p.GIn2407Serfs*36 & Cl1411784 & NS & 16 \\
\hline 7 & Exon 34 & C. $7219 C>T$ & p.Gln2407* & CM136743 & NS & 5 \\
\hline 8 & Exon 34 & c.7229dupA & p.Asn2410Lysfs*33 & Cl130147 & NS & 18 \\
\hline 9 & Exon 34 & c.7275_7276delAC & p.Pro2426Thrfs*16 & CD136755 & NS & 5 \\
\hline 10 & Exon 34 & c. $7303 C>T$ & p.Arg2435* & CM121173 & rs199469465 & 3 \\
\hline 11 & Exon 34 & c.7316dupC & p.Ala2440Serfs*3 & Cl121176 & rs199469468 & 3 \\
\hline 12 & Exon 34 & c. $7330 C>T$ & p.Arg2444* & CM121172 & rs199469464 & 3 , this study \\
\hline 13 & Exon 34 & c.7374dupT & p.Pro2459Serfs*84 & Cl136747 & NS & 5 \\
\hline 14 & Exon 34 & c.7376delC & p.Pro2459Leufs*16 & CD136745 & NS & 5 \\
\hline 15 & Exon 34 & c.7394delC & p.Pro2465Glnfs*10 & CD189225 & NS & 19 \\
\hline 16 & Exon 34 & c.7395delA & p.Val2466Tyrfs*9 & CD1411783 & NS & 16 \\
\hline 17 & Exon 34 & c.7534_7535insAA & p.Thr2512Lysfs*11 & Cl136748 & NS & 5 \\
\hline 18 & Exon 34 & c.7549delC & p.GIn2517Lysfs*5 & CD121174 & rs199469466 & 3 \\
\hline 19 & Exon 34 & c.7732dupT & p.Ser2578Phefs*6 & Cl1815570 & NS & 12 \\
\hline 20 & Exon 34 & c.7736_7737delTT & p.Leu2579Argfs*4 & CD130146 & NS & 18 \\
\hline 21 & Exon 34 & c.7851dupC & p.Asn2618GInfs*12 & Cl136754 & NS & 5 \\
\hline 22 & Exon 34 & c.7863dupG & p.Gln2622Alafs & Cl131140 & rs587776938 & 18 \\
\hline 23 & Exon 34 & c. $8242 C>T$ & p.Arg2748* & CM136744 & rs1085307899 & 5 \\
\hline
\end{tabular}

dbSNP, The Single Nucleotide Polymorphism Database.

${ }^{\dagger}$ HGMD (http://www.hgmd.cf.ac.uk) was accessed on January 10, 2019. NS, not assigned.

Table 2. A summary of case reports of Floating-Harbor syndrome that included growth hormone treatment

\begin{tabular}{|c|c|c|c|c|c|}
\hline Variable & White et al. ${ }^{6}$ & Garcia et al. ${ }^{13)}$ & Nagasaki et al. & Galli-tsinopoulou et al. ${ }^{8)}$ & Present case \\
\hline Country & Australia & Netherland & Japan & Greece & Korea \\
\hline No. of patients (M/F) & $7(6 / 1)$ & $1(0 / 1)$ & $1(1 / 0)$ & $1(0 / 1)$ & $1(0 / 1)$ \\
\hline Age at start (yr) & 4.7 & 9 & 10 & 5.4 & 8 \\
\hline Growth hormone deficiency & - & - & - & + & - \\
\hline Height SDS at start & N/A & -3.11 & -4.9 & -3.8 & -3.33 \\
\hline GH dose (mg/kg/day) & N/A & 0.025 & 0.03 & 0.035 & 0.05 \\
\hline Duration of GH therapy (yr) & 5.5 & 3.5 & 2 & 2 & 4.5 \\
\hline $\mathrm{GV}(\mathrm{cm} / \mathrm{yr})$ & N/A & 8.5 & 5 & 8 & 6.2 \\
\hline Height SDS at last F/U & -2 & -2.4 & -3.6 & -2.2 & -2.7 \\
\hline
\end{tabular}

SDS, standard deviation score; GH, growth hormone; GV, growth velocity; F/U, follow-up. 
therapy and serum IGF-1 level (upper limit of the normal level during therapy). To date, over 100 FHS cases have been reported worldwide, and only a few of these patients were GH deficient. ${ }^{8,14)}$ Table 2 summarizes the results of previous studies on GH therapy in FHS patients. According to these studies, the modalities of GH therapy, age of onset, dose, treatment period, and results have been inconsistent. ${ }^{4,6,8,13)}$

In our case, the patient had normal IGF-1 level and no GH deficiency. After 55 months of GH therapy, her height SDS increased from -3.33 to -2.70 SDS, and her growth velocity was $6.2 \mathrm{~cm} / \mathrm{yr}$. Her pubertal onset and progression were not accelerated. However, her bone age progressed rapidly after puberty. In FHS, a remarkably accelerated bone age after GH therapy was previously reported. ${ }^{4,6}$ At present, it is not known whether bone age acceleration is part of the natural course of FHS or is an adverse effect of GH therapy.

Patients with FHS manifest not only delayed bone age, but also hand abnormalities, including middle phalangeal hypoplasia, hypoplastic metacarpals, absence of a lunate, and a hypoplastic scaphoid. These findings may reflect dysregulation of chondrocyte development. Nagasaki et al. ${ }^{4)}$ suggested that SRCAP mutations can cause skeletal abnormalities due to impaired cAMP-mediated G protein-coupled receptor signaling.

In conclusion, we herein reported the clinical and molecular analysis of a 7-year-old girl with FHS and a heterozygous mutation, c.7330C $>\mathrm{T}$ (p.Arg2444*), in SRCAP. The patient exhibited dysmorphic facial features, severe intellectual disabilities, obsessive-compulsive and aggressive behaviors, and short stature without GH deficiency. Her height SDS was improved after 55 months of GH therapy.

\section{Ethical statement}

Written informed consent was obtained from the parents of the child for publication of this case report.

\section{Conflict of interest}

No potential conflict of interest relevant to this article was reported.

\section{References}

1. Hood RL, Schenkel LC, Nikkel SM, Ainsworth PJ, Pare G, Boycott KM, et al. The defining DNA methylation signature of Floating-Harbor Syndrome. Sci Rep 2016;6:38803.

2. Hood RL, Lines MA, Nikkel SM, Schwartzentruber J, Beaulieu C, Nowaczyk MJ, et al. Mutations in SRCAP, encoding SNF2-related CREBBP activator protein, cause Floating-Harbor syndrome. Am J Hum Genet 2012;90:30813.

3. Leisti J, Hollister DW, Rimoin DL. The Floating-Harbor syndrome. Birth Defects Orig Artic Ser 1975;11:305.
4. Nagasaki K, Asami T, Sato H, Ogawa Y, Kikuchi T, Saitoh A, et al. Long-term follow-up study for a patient with FloatingHarbor syndrome due to a hotspot SRCAP mutation. Am J Med Genet A 2014;164A:731-5.

5. Nikkel SM, Dauber A, de Munnik S, Connolly M, Hood RL, Caluseriu O, et al. The phenotype of Floating-Harbor syndrome: clinical characterization of 52 individuals with mutations in exon 34 of SRCAP. Orphanet J Rare Dis 2013;8:63.

6. White SM, Morgan A, Da Costa A, Lacombe D, Knight SJ, Houlston R, et al. The phenotype of Floating-Harbor syndrome in 10 patients. Am J Med Genet A 2010;152A: 821-9.

7. Arpin S, Afenjar A, Dubern B, Toutain A, Cabrol S, Héron D. Floating-Harbor Syndrome: report on a case in a mother and daughter, further evidence of autosomal dominant inheritance. Clin Dysmorphol 2012;21:11-4.

8. Galli-Tsinopoulou A, Kyrgios I, Emmanouilidou E, Maggana I, Kotanidou E, Kokka P, et al. Growth hormone deficiency: an unusual presentation of floating harbor syndrome. Hormones (Athens) 2011;10:236-40.

9. Seo GH, Park JY, Kim SH, Lee JS, Oh Arum, Lee Y, et al. High diagnostic yield and clinical utility of WES for patients with undiagnosed genetic disorder by automating variant interpretation. BioRxiv 2019 May 9. https://doi. org/10.1101/628438.

10. Johnston H, Kneer J, Chackalaparampil I, Yaciuk P, Chrivia J. Identification of a novel SNF2/SWI2 protein family member, SRCAP, which interacts with CREB-binding protein. J Biol Chem 1999;274:16370-6.

11. Budisteanu M, Bögershausen N, Papuc SM, Moosa S, Thoenes M, Riga D, et al. Floating-Harbor syndrome: presentation of the first Romanian patient with a SRCAP mutation and review of the literature. Balkan J Med Genet 2018;21:83-6.

12. Choi EM, Lee DH, Kang SJ, Shim YJ, Kim HS, Kim JS, et al. The first Korean case with Floating-Harbor syndrome with a novel SRCAP mutation diagnosed by targeted exome sequencing. Korean J Pediatr 2018;61:403-6.

13. García RJ, Kant SG, Wit JM, Mericq V. Clinical and genetic characteristics and effects of long-term growth hormone therapy in a girl with Floating-Harbor syndrome. J Pediatr Endocrinol Metab 2012;25:207-12.

14. Zhang S, Chen S, Qin H, Yuan H, Pi Y, Yang Y, et al. Novel genotypes and phenotypes among Chinese patients with Floating-Harbor syndrome. Orphanet J Rare Dis 2019;14:144.

15. Alfares A, Alfadhel M, Wani T, Alsahli S, Alluhaydan I, $\mathrm{Al}$ Mutairi F, et al. A multicenter clinical exome study in unselected cohorts from a consanguineous population of Saudi Arabia demonstrated a high diagnostic yield. Mol Genet Metab 2017;121:91-5.

16. Seifert W, Meinecke P, Krüger G, Rossier E, Heinritz W, Wüsthof A, et al. Expanded spectrum of exon 33 and 34 mutations in SRCAP and follow-up in patients with 
Floating-Harbor syndrome. BMC Med Genet 2014;15:127.

17. Kehrer M, Beckmann A, Wyduba J, Finckh U, Dufke A, Gaiser U, et al. Floating-Harbor syndrome: SRCAP mutations are not restricted to exon 34. Clin Genet 2014;85:498-9.

18. Le Goff C, Mahaut C, Bottani A, Doray B, Goldenberg A,
Moncla A, et al. Not all floating-harbor syndrome cases are due to mutations in exon 34 of SRCAP. Hum Mutat 2013;34:88-92.

19. Milani D, Scuvera G, Gatti M, Tolva G, Bonarrigo F, Esposito S, et al. Perthes disease: a new finding in FloatingHarbor syndrome. Am J Med Genet A 2018;176:703-6. 